\title{
TOPOLOGICAL AND POLYNOMIAL INVARIANTS, MODULI SPACES, IN CLASSIFICATION PROBLEMS OF POLYNOMIAL VECTOR FIELDS
}

\author{
DANA SCHLOMIUK \\ Dedicated to J. Llibre on his 60th birthday
}

\begin{abstract}
We describe the origin and evolution of ideas on topological and polynomial invariants and their interaction, in problems of classification of polynomial vector fields. The concept of moduli space is discussed in the last section and we indicate its value in understanding the dynamics of families of such systems. Our interest here is in the concepts and the way they interact in the process of topologically classifying polynomial vector fields. We survey the literature giving an ample list of references and we illustrate the ideas on the testing ground of families of quadratic vector fields. In particular, the role of polynomial invariants is illustrated in the proof of our theorem in the section next to last. These concepts have proven their worth in a number of classification results, among them the most recent work on the geometric classification of the whole class of quadratic vector fields, according to their configurations of infinite singularities. An analog work including both finite and infinite singularities of the whole quadratic class, joint work with J. C. Artés, J. Llibre, and N. Vulpe, is in progress.
\end{abstract}

2010 Mathematics Subject Classification: 58K30, 34A26, 34C05, 34C40.

Key words: Quadratic vector fields, phase portrait, affine invariant polynomials, topological invariants, moduli space.

\section{Introduction}

In this article we consider real autonomous differential systems

$$
\frac{d x}{d t}=p(x, y), \quad \frac{d y}{d t}=q(x, y),
$$

where $p, q \in \mathbb{R}[x, y]$, i.e. $p, q$ are polynomials in $x, y$ over $\mathbb{R}$ and their associated vector fields

$$
\widetilde{D}=p(x, y) \frac{\partial}{\partial x}+q(x, y) \frac{\partial}{\partial y} .
$$

Work supported by NSERC. 
We call degree of a system (S) (or of a vector field (1.1)) the integer $n=\max (\operatorname{deg} p, \operatorname{deg} q)$. In particular we call quadratic, respectively cubic, a differential system $(\mathrm{S})$ with $n=2$, respectively $n=3$, and we denote by QS the class of all quadratic systems. We denote by CS the family of all cubic differential systems.

Such systems occur in many areas of applied mathematics which in part motivates the study of these differential systems. On the other hand they are also interesting for theoretical reasons. Indeed, hard problems on polynomial differential systems, among them Hilbert's 16th problem, have been open for more than a century even for the quadratic case. Two other problems on such systems were formulated over 120 years ago. These are the problem of algebraic integrability of such systems (1891) and the problem of the center (1885) which so far was only solved for the quadratic case. Both problems were stated by H. Poincaré.

These problems are of a global nature and while the global study of the whole quadratic class is not within reach at this time, quite a few specific subfamilies of QS and some subfamilies of CS have been successfully studied globally.

While elsewhere in mathematics invariants play a major role in classification problems, many articles on classification problems on polynomial vector fields are rather oblivious of invariants.

The use of polynomial invariants in such classification problems was firstly shown by the Chişinău school founded by C. S. Sibirsky. Inspired by Hilbert's work on the classical theory of invariants for $n$-forms, Sibirsky and his students developed a theory of invariants for polynomial vector fields. In [45] the former student of Sibirsky, N. Vulpe, and his student I. V. Nikolaev gave the classification of all possible configurations of singularities at infinity for the whole quadratic class QS, for the first time in terms of invariant polynomials.

Independently, the author and Pal studied globally the singularities at infinity of the class QWF of quadratic systems possessing a weak focus. The author's work together with Pal was based on a specific normal form of the systems and the classification was done in terms of algebraic equalities and inequalities on these coefficients. While Vulpe and Nikolaev used some artificially defined polynomial invariants which were rather cumbersome and no geometry was used to help explain them, in [53] Schlomiuk and Pal introduced some useful geometric global concepts which helped in understanding their classification. Their work however was not readily applicable to another normal form for the systems because their work was not done in terms of invariants. The authors 
realized that only a fusion of the two methods would lead to an understandable global geometric classification done in terms invariants and hence not bound to a fixed normal form.

Thanks to the AUPELF program as well as to the help of CRM funds, four people from the Chişinău school including Vulpe travelled to Montreal in 2000. This resulted in a collaboration of the author with Vulpe who combined in [55] the two approaches: of global geometrical concepts such as for example multiplicity divisors on the plane or on the line at infinity in [53], and invariant polynomials such as they were used in [55]. Due to the geometry introduced in [53], Vulpe and the author simplified in [55] the invariants used in [45] so that the final classification in terms of invariant polynomials became more transparent.

Much work on geometric classifications of families of polynomial vector fields followed, some done by the author in collaboration with Vulpe $([\mathbf{5 4}],[\mathbf{5 6}],[\mathbf{5 7}],[\mathbf{5 8}],[\mathbf{5 9}],[\mathbf{6 1}])$, other work done by Vulpe together with Artés and Llibre $([\mathbf{7}],[\mathbf{9}])$ or by the author together with Artés, Llibre, and Vulpe $([\mathbf{6}])$.

The first goal of this article is to trace the evolution of ideas in classification problems of families of polynomial vector fields, to clarify the essential role played by topological and polynomial invariants in these problems and to show how the interplay between the topological and polynomial invariants provides us with a solid understanding of the basic properties of the families we want to classify. Another goal is to give a very succinct critical survey of the results on classification problems of planar polynomial vector fields. We illustrate in our last sections the significance of introducing moduli spaces in this area of research. Our aim was also to write in a self-contained manner, as much as possible, so as to make this article accessible to anyone interested in these problems, to encourage new work to be done by people not necessarily belonging to the Sibirsky school, or to the author's collaborators. With the exception of the proof of the theorem in the section on bifurcations, where we had to rely on results in $[\mathbf{5 9}]$ in order to show how invariant polynomials work, we think this aim was attained.

After a short introduction, we describe in Section 2 the role of invariants in mathematical classification problems. In Section 3 we consider classification problems of families of polynomial vector fields and introduce the necessary notions. In this section we survey the results on classification problems of families of low degree polynomial systems, describe the significant role played by topological and polynomial invariants in classifications problems on planar polynomial vector fields, as 
well as how they interact. We also show the role of polynomial invariants in constructing bifurcation diagrams independent of normal forms and raise questions leading us to moduli spaces in these global classification problems.

\section{Mathematical classification problems and the role of invariants}

2.1. Basic concepts. In a classification problem one starts with a set of objects $X$ and an equivalence relation $\sim$ on $X$. To solve the classification problem of the objects of $X$ with respect to $\sim$ means to present a complete list of representatives of all equivalence classes of $X$ which is minimal, that is such that no equivalence class is represented twice in the list or to put it differently, any two representatives in the list are non-equivalent.

We emphasize here the importance of this second part of solving a classification problem. Since objects could be quite complex, without presenting a proof that the objects in the list are distinct, we may end up with repetitions, without being able to see them due to the complexity of the objects.

In a mathematical classification problem the elements of $X$ are mathematical objects such as for example algebraic curves, topological spaces, smooth manifolds, etc. In topology, one of the equivalence relations which is considered is the homeomorphism of two topological spaces, and the main problem is to establish if two given topological spaces $X, Y$ are homeomorphic or not. To see that they are, it suffices to construct a homeomorphism $f: X \rightarrow Y$. It is clearly much harder to prove that they are not homeomorphic since this would mean proving that any bijection $g: X \rightarrow Y$ is not a homeomorphism.

Given a set $X$ endowed with an equivalence relation $\sim$, an invariant on $X$ with respect to $\sim$ is a map $I: X \rightarrow Y$ such that if $x_{1} \sim x_{2}$ then $I\left(x_{1}\right)=I\left(x_{2}\right)$. In proving non-equivalence, a major role is played by invariants. Indeed, to show that two elements $a, b$ of $X$ are not equivalent, it then suffices to find and invariant $I$ on $X$ with respect to $\sim$ such that $I(a) \neq I(b)$.

Given a set $X$ endowed with an equivalence relation $\sim$, we can form the quotient set $X / \sim$ of equivalence classes $[a]=\{b \mid b \sim a\}$ of $X$. The canonical map $X \rightarrow X / \sim$ is clearly an invariant, the trivial invariant, and any other invariant on $X$ factors through this map. 
In many problems the equivalence is induced by a group action on $X$. By this we mean firstly that we have a group $G$ and a map $A$ : $G \times X \rightarrow X$ which respects the group law of $G$. We denote by $g x$ the element $A(g, x)$ and we ask that i) $g_{2}\left(g_{1} x\right)=\left(g_{2} g_{1}\right) x$ for every $g_{1}, g_{2} \in G$ and for every $x \in X$, and that ii) $e x=x$ for every $x$ in $X, e$ being the neutral element of $G$. This is called a left group action. We say that two elements $a, b$ of $X$ are equivalent with respect to the group action of $G$ on $X$ if and only if there exists an element $g$ of $G$ such that $b=g a$. In this case we say that we classify $X$ with respect to the group action. The equivalence class $[a]$ of $a$ is in this case called the orbit of $a$ under the action of $G$.

The set $Y$ for an invariant $I: X \rightarrow Y$ could be a set of numbers. For example the map associating to every ellipse its largest semi-axis is an invariant with respect to the action of the group of rigid motions (see Example 1 below) and $Y=\mathbb{R}$ in this case. Here we have a very simple example of an invariant. But in general the construction of invariants could involve much more complicated mathematical objects. For example this is the case for S. Donaldson's invariants used to distinguish between different smooth differential structures on 4-dimensional topological manifolds (see $[\mathbf{3 4}]$ ). For this work Donaldson was awarded the Fields medal in 1986.

By a complete set of invariants we understand a set of invariants $I_{j}: X \rightarrow Y_{j}, j \in J$ with respect to $(X, \sim)$ such that $a \sim b$ if and only if for every $j \in J$ we have $I_{j}(a)=I_{j}(b)$.

\subsection{Some examples.}

Example 1. The group of rigid transformations of the plane acts on the set of all conic curves. We recall that by a rigid transformations we mean a map $T: \mathbb{R}^{2} \rightarrow \mathbb{R}^{2}$ which conserves distances. It is well known that each such transformation is a combination of rotations, reflections and translations. For every rigid motion $M$ and conic $C$, we get the conic $M C$ obtained after applying to $C$ the rigid motion $M$. A set of representatives of the classification of real non-empty conics with respect to this group action is formed by all ellipses $x^{2} / a^{2}+y^{2} / b^{2}=1$, all hyperbolas $x^{2} / a^{2}-y^{2} / b^{2}=1$, all parabolas $y-k x^{2}=0$, all reducible conics $x(y-k x)=0, x(x-a)=0, x^{2}=0, x^{2}+y^{2}=0$ with $a, b, c>0$.

Example 2. Let $\operatorname{Aff}(2, \mathbb{R})$ be the group of affine transformations of the plane. Then this group acts on the set $X$ of real, non-empty conics too. A set of representatives of this group action on $X$ is formed by 
the following 6 conics: $x^{2}+y^{2}=1, x^{2}-y^{2}=1, y-x^{2}=0, x y=0$, $x(x-1)=0$ and $x^{2}+y^{2}=0$.

Observation. We note that as the group of transformations acting on a set $X$ becomes larger, the orbits become larger which results in fewer equivalence classes.

Example 3. On the set of real, non-empty projective conics acts the group $\mathrm{PGL}(3, \mathbb{R})$ of all projective transformations on the projective plane $\mathbb{P R}^{2}$. A set of representatives is formed by the conic $Z^{2}-X Y=0$ and the reducible conics $X Y=0, X(X-Z)=0, X^{2}=0$ and $X^{2}+Y^{2}=0$.

Example 4. Consider the set of two-dimensional connected, compact, orientable, topological manifolds. The topological classification of these manifolds with respect to the homeomorphism equivalence relation is given by the following theorem:

Every compact, connected, orientable two-dimensional topological manifold is a sphere with $g$ handles.

Here the number $g$ of handles is a topological invariant, the number of holes of the compact surface. The singleton $\{g\}$ is a complete set of invariants. Apparently this classification was known since the 19-th century. A proof can be found in the book of Seifert and Threlfall [63].

Example 5. A greatly more complicated example is the classification of smooth, compact, connected, three-dimensional manifolds which was only recently completed by G. Perelman based on the geometrization conjecture of W. Thurston and on work done by R. Hamilton on the Ricci flow (see $[\mathbf{6 5}]$ ). The proof of this conjecture also solves the problem of Poincaré that any 3-dimensional compact topological manifold for which any loop can be continuously deformed to a point is homeomorphic to the 3-dimensional sphere.

The problem of listing all smooth structures on four-dimensional topological manifolds is open. It is known that some four-dimensional topological manifolds do not admit smooth structures (see [65]).

Other examples of classification problems which were solved are: the classification of compact Riemann surfaces with respect to biholomorphical equivalence relation and the classification of finite simple groups with respect to isomorphism. 


\section{Classification problems on planar polynomial vector fields}

3.1. A general discussion. Let $X$ be a specific family of planar polynomial vector fields which we consider equipped with the topological equivalence relation, defined as follows:

Two polynomial differential systems $S_{1}$ and $S_{2}$ are topologically equivalent if and only if there exists a homeomorphism of the plane carrying the oriented phase curves of $S_{1}$ to the oriented phase curves of $S_{2}$ and preserving the orientation.

To every polynomial differential system we associate its phase portrait which is the partition of the plane into oriented phase curves. Clearly if we reverse the time we get the same phase curves but with reversed orientation. When listing all phase portraits of a given family of systems, it is then convenient to list only one of the two portraits which both induce the same foliation with singularities. By reversing the orientation, another phase portrait of the family is obtained. For this reason sometimes authors prefer to use the following equivalence relation:

Two polynomial differential systems $S_{1}$ and $S_{2}$ are topologically equivalent if and only if there exists a homeomorphism of the plane carrying the phase curves of $S_{1}$ to the phase curves of $S_{2}$ and preserving or reversing (globally) the orientation.

The first definition is the one commonly used and it is in some sense the better notion as it preserves stability, or instability, of singularities. But people working on classifying systems belonging to specific families prefer the second notion because it cuts in half the number of phase portraits to be exhibited.

The first successful articles on classifying families of planar polynomial systems were done by Sibirsky and Vulpe, one published in 1975 [74] and another in 1977 [75]. A few unsuccessful attempts to classify quadratic systems with a center were done before 1975 (see [52] for a history of this problem and for references). Vulpe's article [69] published in 1983 contained the first correct classification for this important class of all quadratic systems with a center. Vulpe not only listed there all 31 phase portraits of this family but also gave necessary and sufficient conditions in terms of polynomial invariants for the realization of each one of the phase portraits, when the center is placed at the origin. 
Thirty years have passed since the publication of this article and in this period of time over 50 articles appeared solving classification problems for quadratic or cubic differential systems. These articles are of two kinds: those listing phase portraits but without proving with the help of topological invariants that the lists contain topologically distinct phase portraits and without characterizing the phase portraits in terms of polynomial invariants; those proving that the phase portraits are indeed distinct and characterizing each phase portrait in terms of polynomial invariants.

Strictly speaking the articles do not actually list phase portraits since these would be impossible to draw, containing all oriented phase curves. Instead the articles list the separatrix configurations as defined by Markus in $[\mathbf{4 3}]$. But giving the separatrix configuration suffices since from it the corresponding phase portrait is immediately grasped so that we can actually say that the phase portraits are listed.

By a separatrix configuration of a $C^{1}$ planar differential system we understand a collection of curves consisting of separatrices and one oriented phase curve from each canonical region.

The separatrix configuration is a topological invariant, i.e. it is an invariant with respect to the topological equivalence relation.

A separatrix configuration could contain many curves which makes it sometimes hard to see in some cases if two separatrix configurations are or not homeomorphic. Finer topological invariants are necessary for this. As we mentioned in the preceding section, to solve a classification problem means to present a list of representatives for which a proof is given that any two objects in the list are not equivalent. Since some of the articles do not explicitly prove that the phase portraits are in fact non-equivalent, occasionally, in the lists of phase portraits a separatrix configuration appears listed twice. Sometimes Erratum or Corrigendum papers appeared adjusting the results. Thus the classification articles fall in two categories: A) those using only the separatrix configuration invariant and without proofs that the lists presented contain only nonequivalent objects and B) those using finer invariants (topological or algebraic) with the help of which proofs are given that the lists of representatives are minimal, i.e. contain only non-equivalent objects.

The classifications are based on specific normal forms and they are done in terms of equalities and inequalities expressed in terms of the coefficients of these normal forms which are a basic tool for obtaining these classifications. They are so chosen as to be well adapted for the 
specific families we want to classify. In general, for studying globally a family of systems, more than just one normal form is usually needed. Thus there arises the problem of gluing two such normal forms. But this gluing is not always done. Some authors just list portraits according to the specific normal forms which results in listing twice or even more times the same phase portrait, once for each one of the normal forms, in case the domains of definition of these normal forms overlap (see for example $[\mathbf{4 8}],[\mathbf{1 7}]$ ) so that we end up having numerous lists. Transferring the results from one normal form to another is usually not done and the results are stated in terms of the coefficients of the various fixed normal forms. When our studies are done in invariant form, i.e. are independent of the normal forms in which the systems are presented, the results obtained from distinct normal forms are integrated. While some topological invariants are difficult if not even impossible to compute, others such as the polynomial invariants (see Subsection 3.4) can easily be computed allowing us to glue results. During the past twelve years collaborations of the Chişinău school founded by Sibirsky, the Barcelona school and the author was made possible and classifications where both geometric methods and algebraic ones involving polynomial invariants were obtained and this kind of work is now in full progress.

\subsection{Brief survey of results on classification problems of low} degree polynomial differential systems. In topology we first had the theorem classifying compact connected orientable two-dimensional topological manifolds. These surfaces admit only one smooth structure so that this also gives the classification of their two-dimensional smooth structures. The analog theorem for three-dimensional smooth manifolds was only recently proved by Perelman (see [65]). In the four-dimensional case the problem is open.

Turning now to classification problems of planar polynomial vector fields, naturally the first cases considered were the ones on the lowest degrees, that is the quadratic and cubic differential systems. The problem of classifying topologically the whole quadratic class QS is a very hard one, still open today. There is a tendency to look upon quadratic system as being very simple which they are indeed, being the simplest ones apart from the linear ones. But going too far in this kind of evaluation could be very treacherous and leading to errors. This occurs when one uses the term "simple" thinking in fact about the whole quadratic class or one of its large subfamily. These are in general very hard classifying problems, especially so for the whole quadratic family. The class QS depends on five parameters modulo the action of the group of affine transformations 
and time rescaling. This large number of parameters explains only in part why this problem is so hard. The problem is difficult also because of the elusive nature of limit cycles. But even when we leave aside the limit cycles, by considering the problem of classifying QS modulo limit cycles (that is when all limit cycles around one singularity are collapsed to that singularity), this problem is still open today.

It is expected that the topological classification of the family of all quadratic systems would lead to a finite number of phase portraits estimated to be over two thousand.

The earliest (correct) work was done for quadratic homogeneous systems, for which the polynomials $p, q$ are quadratic homogeneous polynomials (see [74], [75]). These articles were followed by numerous others among them those we mention below. We do not claim that our lists are complete but they contain the articles published by the Chişinău and Barcelona schools and by myself, alone or with collaborators. As in this work we are interested in invariants and their use in classification problems, we single out the classification articles which do not use other invariants than the phase portraits (category A) defined earlier) from those in which finer topological or polynomial invariants are used (category B) defined earlier). We first list below the families classified in papers of the category A) and indicate the corresponding references. Some of these families are defined by a specific property which often involves the number or the nature of the singularities of the systems as we indicate below:

- Quadratic systems with a center, [51], [76], [47].

- Systems without any finite real singularity (these are called chordal systems), [26], [18].

- Systems with a unique finite singularity, $[\mathbf{2 4}]$.

- Systems with a focus and one anti-saddle, $[\mathbf{3}]$.

- Systems with a third order weak focus, [40].

- Quadratic and cubic systems with all points at infinity as singularities, [27].

- Quadratic systems with a higher order singularity with two zero eigenvalues, [31].

Some families are defined as having a Darboux first integral (or a Darboux inverse integrating factor) having one of the following properties:

- A rational first integral, $[\mathbf{3 8}],[\mathbf{3 9}],[\mathbf{3 6}]$.

- A polynomial first integral, [25].

- A rational first integral of degree 2 or rational first integral of degree 3, [15], [16], [38], [39]. 
- Quadratic systems with a polynomial inverse integrating factor, $[\mathbf{2 2}]$.

Other families are defined by one of the following properties:

- Quadratic Hamiltonian systems, [2], [4].

- Bounded quadratic systems, $[\mathbf{2 3}],[\mathbf{3 5}]$.

- Reversible Darboux integrable quadratic systems, [37].

- Cubic systems which are defined by homogeneous polynomials, $[\mathbf{2 1}]$.

- Cubic systems with homogeneous nonlinearities, [14].

- The reduced cubic Kukles systems with a center, [50].

- Cubic systems with canonical period annuli, [19].

- Cubic systems symmetric with respect to a center, [49].

- A class of reversible cubic systems with centers, [20].

- Quadratic systems which are structurally stable modulo limit cycles, i.e. limit cycles around one singularity are collapsed to that singularity, [1].

- Structurally stable quadratic foliations, $[\mathbf{3 2}]$.

- Semi-linear quadratic systems, i.e. one equation is defined by a polynomial of the form $a x+b y,[\mathbf{4 1}]$.

All papers in the category B) not only give the lists of phase portraits but also for each phase portrait, invariant necessary and sufficient conditions are given for its realization. The fact that these conditions are expressed in invariant form means that no matter how a system is presented, one can easily check if this system belongs to the family and if it does, then what is its phase portrait. We indicate below the references according to the specific properties defining the families.

Families of quadratic or cubic systems defined by properties involving singularities:

- Quadratic systems with a center, [69].

- Quadratic systems with an integrable saddle, [8].

- Quadratic systems with a symmetry center and simple infinite singular points, $[\mathbf{4 2}]$.

- Quadratic systems with a unique finite singular point of multiplicity two, possessing two zero eigenvalues, [44].

- Quadratic systems with the line at infinity filled up with singularities, $[\mathbf{5 9}]$.

- Quadratic systems without finite singularities, [67].

- Quadratic systems with a single finite singularity which in addition is simple, $[\mathbf{6 8}]$. 
- Quadratic systems with a finite singular point of multiplicity 4, $[73],[66]$.

- Quadratic systems with a singular point of multiplicity 3, [72].

Other families are defined by one of the following properties:

- Quadratic Hamiltonian systems, [33].

- Quadratic systems with invariant lines of total multiplicity greater than or equal to four, $[\mathbf{5 6}],[\mathbf{5 8}],[\mathbf{6 0}],[\mathbf{5 4}],[\mathbf{5 7}]$.

- Quadratic Lotka-Volterra differential systems, [61], [62].

- Quadratic systems of Darboux type, [71].

- Homogeneous quadratic systems, [74], [75].

In this second category B) of systems we also have classifications of the whole quadratic class according to two equivalence relations:

- The topological equivalence in neighborhoods of infinity of two quadratic systems, [45], [55].

- The geometrical equivalence of two quadratic systems according to their singularities at infinity, [6].

The geometrical equivalence relation is finer than the topological one, i.e. if two systems are geometrically equivalent according to their singularities at infinity, they are also topological equivalent in neighborhoods of infinity.

3.3. Topological invariants. Up to now we mentioned only one topological invariant, namely the separatrix configuration. If a family of systems has only a small number of phase portraits and these are not very complicated then this invariant is sufficient. For example for the set QW3 of all quadratic differential systems possessing a third order weak focus $[\mathbf{4 0}]$ we found only 18 distinct phase portraits which are all rather simple and we can easily see that they are distinct just by looking at their separatrix configurations. If the phase portraits are numerous and more complex, to distinguish phase portraits by using the separatrix configuration invariant is more difficult. It is important in this case to use simpler invariants to distinguish phase portraits. Simpler topological invariants were used for example in $[\mathbf{6 2}]$. We indicate below some of these simpler invariants.

I. Singularities, invariant lines, multiplicities and indices:

- $\mathcal{N}=$ total number of all singularities of the systems;

- $\left(\begin{array}{c}\mathcal{N}_{f} \\ \mathcal{T}_{m}\end{array}\right)=$ the number $\mathcal{N}_{f}$ of all distinct finite singularities having a total multiplicity $\mathcal{T}_{m}$;

- $\operatorname{deg} J=$ the sum of the indices of all finite singularities of the systems; 
- $\mathcal{N}_{A I L}^{\text {sing }}=$ total number of affine invariant lines filled up with singularities;

- $\mathcal{N}_{\infty}=$ total number of infinite distinct singularities.

II. Connections of separatrices:

- $\# S C_{s}^{s}=$ total number of connections of a finite saddle to a finite saddle;

- \#SC$C_{s}^{S}=$ total number of connections of a finite saddle to an infinite saddle;

- \#SC$C_{s}^{\mathcal{S N}}=$ total number of connections of a finite saddle to an infinite saddle-node;

- $\# S C_{s n}^{s}=$ total number of connections of a finite saddle-node to a finite saddle;

- $\# S C_{s n}^{S}=$ total number of connections of a finite saddle-node to an infinite saddle;

- $\# S C_{s n}^{\mathcal{S N}}=$ total number of connections of a finite saddle-node to an infinite saddle-node;

- $\# S C_{s n(h h)}^{\mathcal{S}}=$ total number of separatrices dividing the two hyperbolic sectors of finite saddle-nodes, going to infinite saddles;

- $\# S C_{s n(h h)}^{\mathcal{S N}}=$ total number of separatrices dividing the two hyperbolic sectors of finite saddle-nodes connecting with separatrices of infinite saddle-nodes;

- \# $\operatorname{Sep}_{(H H)}^{\mathcal{S N}}=$ total number of separatrices of infinite saddlenodes located in the affine part of the plane and dividing the two hyperbolic sectors.

III. The number of separatrices or orbits leaving from or ending at a singular point:

- $M_{\text {sep }}^{\widetilde{n}}=\max \{\operatorname{sep}(\widetilde{n}) \mid \widetilde{n}$ is a node $\}$, where $\operatorname{sep}(\widetilde{n})$ is the number of separatrices leaving from or ending at a finite node $\widetilde{n}$;

- $M_{\text {sep }}^{\widetilde{s} \tilde{n}}=\max \{\operatorname{sep}(\widetilde{s} \tilde{n}) \mid \widetilde{s n}$ is a node $\}$, where $\operatorname{sep}(\widetilde{s} \tilde{})$ is the number of separatrices leaving from or ending at a finite saddlenode $\widetilde{s n}$;

- $M_{\text {orb }}=\max \{\operatorname{orb}(p) \mid p$ is a finite $\operatorname{singularity}\}$, where orb $(p)$ is the number of orbits leaving from or arriving at $p$;

- $M_{\mathrm{ORB}}=\max \left\{\operatorname{orb}\left(p_{1}, p_{2}\right) \mid p_{1}, p_{2}\right.$ are infinite singularities $\}$, where orb $\left(p_{1}, p_{2}\right)$ is the number of orbits connecting $p_{1}$ with $p_{2}$.

Simpler topological invariants are very useful once separatrix configurations are found, since they allow us to distinguish them. Topological invariants are in general very hard to calculate from the coefficients of 
the systems (for example the topological invariant defined as the number of limit cycles of a polynomial vector field). Only occasionally, using analytic tools, we can calculate the exact number of limit cycles. Polynomial invariants (see the next two sections) can easily be calculated and therefore they are of great help in qualitative studies.

3.4. The affine group action on polynomial vector fields. Consider real planar polynomial differential systems $(\mathrm{S})$. We denote by $\mathbf{P S}$ the set of all planar polynomial systems $(\mathrm{S})$ of a fixed degree $\mathbf{n}$. On the set PS acts (left action) the group $\operatorname{Aff}(2, \mathbb{R})$ of affine transformations on the plane:

$$
\begin{aligned}
\operatorname{Aff}(2, \mathbb{R}) \times \mathbf{P S} & \longrightarrow \mathbf{P S} \\
(g, S) & \longrightarrow \widetilde{S}=g S .
\end{aligned}
$$

This action is defined as follows:

Consider an affine transformation $g \in \operatorname{Aff}(2, \mathbb{R}), g: \mathbb{R}^{2} \longrightarrow \mathbb{R}^{2}$. For this transformation we have:

$$
g:\left(\begin{array}{l}
\widetilde{x} \\
\widetilde{y}
\end{array}\right)=M\left(\begin{array}{l}
x \\
y
\end{array}\right)+B ; \quad g^{-1}:\left(\begin{array}{l}
x \\
y
\end{array}\right)=M^{-1}\left(\begin{array}{l}
\widetilde{x} \\
\widetilde{y}
\end{array}\right)-M^{-1} B,
$$

where $M=\left\|M_{i j}\right\|$ is a $2 \times 2$ nonsingular matrix and $B$ is a $2 \times 1$ matrix over $\mathbb{R}$. For every $S \in \mathbf{P S}$ we can form its induced transformed system $\widetilde{S}=g S$ :

$$
\frac{d \widetilde{x}}{d t}=\widetilde{p}(\widetilde{x}, \widetilde{y}), \quad \frac{d \widetilde{y}}{d t}=\widetilde{q}(\widetilde{x}, \widetilde{y})
$$

where

$$
\left(\begin{array}{l}
\widetilde{p}(\widetilde{x}, \widetilde{y}) \\
\widetilde{q}(\widetilde{x}, \widetilde{y})
\end{array}\right)=M\left(\begin{array}{l}
\left(p \circ g^{-1}\right)(\widetilde{x}, \widetilde{y}) \\
\left(q \circ g^{-1}\right)(\widetilde{x}, \widetilde{y})
\end{array}\right)
$$

The map (3.1) verifies the axioms for a left group action. For every subgroup $G \subseteq \operatorname{Aff}(2, \mathbb{R})$ we have an induced action of $G$ on PS.

Definition 3.1. Consider a subset $\mathcal{A}$ of $\mathbf{P S}$ and a subgroup $G$ of $\operatorname{Aff}(2, \mathbb{R})$. We say that the subset $\mathcal{A}$ is invariant with respect to the group $G$ if for every $g$ in $G$ and for every system $S$ in $\mathcal{A}$ the transformed system $g S$ is also in $\mathcal{A}$.

We can identify the set of systems in PS with a subset of $\mathbb{R}^{m}$ via the embedding PS $\hookrightarrow \mathbb{R}^{m}$ which associates to each system $(\mathrm{S})$ in $\mathbf{P S}$ the $m$-tuple $\left(\mathbf{a}_{00}, \ldots, \mathbf{b}_{0 n}\right)$ of its coefficients. We denote by $\mathbb{R}_{\mathcal{A}}^{m}$ the image of the subset $\mathcal{A}$ of $\mathbf{P S}$ under the embedding $\mathbf{P S} \hookrightarrow \mathbb{R}^{m}$.

For every $g \in \operatorname{Aff}(2, \mathbb{R})$ let $r_{g}: \mathbb{R}^{m} \longrightarrow \mathbb{R}^{m}$ be the map which corresponds to $g$ via this embedding. We know (cf. [64]) that $r_{g}$ is linear and 
that the map $r: \operatorname{Aff}(2, \mathbb{R}) \longrightarrow G L(m, \mathbb{R})$ thus obtained is a group homomorphism. For every subgroup $G$ of $\operatorname{Aff}(2, \mathbb{R}), r$ induces a representation of $G$ onto a subgroup $\mathcal{G}$ of $G L(m, \mathbb{R})$.

The group $\operatorname{Aff}(2, \mathbb{R})$ acts on $\mathbf{Q S}$ and this yields an action of this group on $\mathbb{R}^{12}$. For every subgroup $G$ of $\operatorname{Aff}(2, \mathbb{R}), r$ induces a representation of $G$ onto a subgroup $\mathcal{G}$ of $G L(12, \mathbb{R})$.

This group action yields an equivalence relation on $\mathbf{P S}$, namely two systems $S_{1}, S_{2}$ are affinely equivalent when there is an affine transformation sending $S_{1}$ to $S_{2}$. The orbit $\operatorname{Orb}(S)$ of a system $S$ under the action of the group is the affine equivalence class of $S$.

3.5. Polynomial invariants. A very useful concept is the notion of polynomial invariant. This kind of invariant sends a polynomial differential system with coefficients $a=\left(a_{00}, a_{10}, \ldots, b_{0 n}\right)$ to a polynomial $U$ belonging to the ring $\mathbb{R}\left[a_{00}, a_{10}, \ldots, b_{0 n}, x, y\right]$. If $g$ is an affine transformation and $S_{a}$ is the system with coefficients $a$ then let $g S_{a}$ be the transformed system of $S_{a}$ via $g$ and let us denote its sequence of coefficients $g a$. Then this polynomial $U$ is an invariant with respect to affine equivalence if and only if for every system $S_{a}$ and every transformation $g$ we have: $U(g a, g(x, y))=U(a, x, y)$. This notion however is a too restrictive for classification purposes. Allowing the second term to contain a factor of the form $\operatorname{det}(g)^{-\chi}$, with $\chi \in \mathbb{Z}$, the systems to move in a subset $\mathcal{A}$ and the group to be a subgroup $G$ of the affine group we obtain the more general notion defined below:

Definition 3.2. A polynomial $U(a, x, y) \in \mathbb{R}[a, x, y]$ is called a comitant with respect to $(\mathcal{A}, G)$, where $\mathcal{A}$ is an affine invariant subset of $\mathbf{P S}$ and $G$ is a subgroup of $\operatorname{Aff}(2, \mathbb{R})$, if there exists $\chi \in \mathbb{Z}$ such that for every $(g, \mathbf{a}) \in G \times \mathbb{R}_{\mathcal{A}}^{m}$ the following identity holds in $\mathbb{R}[x, y]$ :

$$
U\left(r_{g}(\mathbf{a}), g(x, y)\right) \equiv(\operatorname{det} g)^{-\chi} U(\mathbf{a}, x, y),
$$

where $\operatorname{det} g=\operatorname{det} M$. If the polynomial $U$ does not explicitly depend on $x$ and $y$ then it is called invariant. The number $\chi \in \mathbb{Z}$ is called the weight of the comitant $U(a, x, y)$. If $G=G L(2, \mathbb{R})$ (or $G=\operatorname{Aff}(2, \mathbb{R})$ ) and $\mathcal{A}=\mathbf{P S}$ then the comitant $U(a, x, y)$ is called $G L$-comitant (respectively, affine comitant).

These comitants will also be called polynomial invariants. In fact these are the polynomial invariants with which we work.

Definition 3.3. A subset $X \subset \mathbb{R}^{m}$ will be called $G$-invariant, if for every $g \in G$ we have $r_{g}(X) \subseteq X$. 
Let $T(2, \mathbb{R})$ be the subgroup of $\operatorname{Aff}(2, \mathbb{R})$ formed by translations. Consider the linear representation of $T(2, \mathbb{R})$ into its corresponding subgroup $\mathcal{T} \subset G L(m, \mathbb{R})$, i.e. for every $\tau \in T(2, \mathbb{R}), \tau: x=\widetilde{x}+\alpha, y=\widetilde{y}+\beta$ we consider as above $r_{\tau}: \mathbb{R}^{m} \longrightarrow \mathbb{R}^{m}$.

Definition 3.4. A comitant $U(a, x, y)$ with respect to $(\mathcal{A}, G)$ is called a $T$-comitant if for every $(\tau, \mathbf{a}) \in T(2, \mathbb{R}) \times \mathbb{R}_{\mathcal{A}}^{m}$ the identity $U\left(r_{\tau}(\mathbf{a}), \tau(x, y)\right)=U(\mathbf{a}, \widetilde{x}, \widetilde{y})$ holds in $\mathbb{R}[\widetilde{x}, \widetilde{y}]$.

Definition 3.5. The polynomial $U(a, x, y) \in \mathbb{R}[a, x, y]$ has well determined sign on $V \subset \mathbb{R}^{m}$ with respect to $x, y$ if for every fixed a $\in V$, the polynomial function $U(\mathbf{a}, x, y)$ is not identically zero on $V$ and has constant sign outside its set of zeroes on $V$.

Observation 3.1. We draw attention to the fact, that if a $T$-comitant $U(a, x, y)$ with respect to $(\mathcal{A}, G)$ of even weight (see Definition 3.2 above) is a binary form in $x, y$, of even degree in the coefficients of $(\mathrm{S})$ and has well determined sign on the affine invariant algebraic subset $\mathbb{R}_{\mathcal{A}}^{m}$ then this property is conserved by any affine transformation and the sign is conserved.

We define here below some polynomial invariants, some of which will be used later.

Consider real quadratic systems, i.e. systems of the form:

$$
\left\{\begin{array}{l}
\dot{x}=p_{0}+p_{1}(a, x, y)+p_{2}(a, x, y) \equiv p(a, x, y), \\
\dot{y}=q_{0}+q_{1}(a, x, y)+q_{2}(a, x, y) \equiv q(a, x, y)
\end{array}\right.
$$

with $\max (\operatorname{deg}(p), \operatorname{deg}(q))=2$ and

$$
\begin{array}{lll}
p_{0}=a_{00}, & p_{1}(a, x, y)=a_{10} x+a_{01} y, & p_{2}(a, x, y)=a_{20} x^{2}+2 a_{11} x y+a_{02} y^{2}, \\
q_{0}=b_{00}, & q_{1}(a, x, y)=b_{10} x+b_{01} y, & q_{2}(a, x, y)=b_{20} x^{2}+2 b_{11} x y+b_{02} y^{2},
\end{array}
$$

where $a=\left(a_{00}, a_{10}, a_{01}, a_{20}, a_{11}, a_{02}, b_{00}, b_{10}, b_{01}, b_{20}, b_{11}, b_{02}\right)$ is the 12 -tuple of the coefficients of an arbitrary system $(\mathrm{S})$ and denote $\mathbb{R}[a, x, y]=$ $\mathbb{R}\left[a_{00}, a_{10}, a_{01}, a_{20}, a_{11}, a_{02}, b_{00}, b_{10}, b_{01}, b_{20}, b_{11}, b_{02}, x, y\right]$.

Notation 3.2 . We denote by $\mathbf{a}=\left(\mathbf{a}_{00}, \mathbf{a}_{10}, \ldots, \mathbf{b}_{02}\right)$ a specific point in $\mathbb{R}^{12}$ and we keep $a_{i j}$ and $b_{i j}$ as parameters. Each particular system (S) yields an ordered 12-tuple a of its coefficients.

Let us consider the polynomials

$$
\begin{array}{ll}
C_{i}(a, x, y)=y p_{i}(a, x, y)-x q_{i}(a, x, y) \in \mathbb{R}[a, x, y], & i=0,1,2 \\
D_{i}(a, x, y)=\frac{\partial}{\partial x} p_{i}(a, x, y)+\frac{\partial}{\partial y} q_{i}(a, x, y) \in \mathbb{R}[a, x, y], & i=1,2
\end{array}
$$


As it was shown in $[\mathbf{6 4}]$ the polynomials

$$
\left\{C_{0}(a, x, y), C_{1}(a, x, y), C_{2}(a, x, y), D_{1}(a), D_{2}(a, x, y)\right\}
$$

of degree one in the coefficients of systems (S) are $G L$-comitants of these systems.

Notation 3.3. Let $f, g \in \mathbb{R}[a, x, y]$ and

$$
(f, g)^{(k)}=\sum_{h=0}^{k}(-1)^{h}\left(\begin{array}{l}
k \\
h
\end{array}\right) \frac{\partial^{k} f}{\partial x^{k-h} \partial y^{h}} \frac{\partial^{k} g}{\partial x^{h} \partial y^{k-h}}
$$

$(f, g)^{(k)} \in \mathbb{R}[a, x, y]$ is called the transvectant of index $k$ of $(f, g)$ (cf. [29], $[46])$.

Theorem 3.1 (see [70]). Any GL-comitant of systems (S) can be constructed from the elements of the set (3.3) by using the operations: +, ,$- \times$, and by applying the differential operation $(*, *)^{(k)}$.

Remark 3.4. We point out that the elements of the set (3.3) generate the whole set of $G L$-comitants and hence also the set of affine comitants as well as of set of the $T$-comitants.

Notation 3.5. Consider the polynomial $\Phi_{\alpha, \beta}=\alpha P+\beta Q \in \mathbb{R}[a, X, Y, Z, \alpha, \beta]$ where $P=Z^{2} p(X / Z, Y / Z), Q=Z^{2} q(X / Z, Y / Z), p, q \in \mathbb{R}[a, x, y]$ and $\max \left(\operatorname{deg}_{(x, y)} p, \operatorname{deg}_{(x, y)} q\right)=2$. Then

$$
\begin{aligned}
\Phi_{\alpha, \beta}=c_{11}(a, & \alpha, \beta) X^{2}+2 c_{12}(a, \alpha, \beta) X Y+c_{22}(a, \alpha, \beta) Y^{2} \\
& +2 c_{13}(a, \alpha, \beta) X Z+2 c_{23}(a, \alpha, \beta) Y Z+c_{33}(a, \alpha, \beta) Z^{2}
\end{aligned}
$$

and we denote

$$
\begin{aligned}
& D(a, x, y)=4\left[\operatorname{det}\left\|c_{i j}(a, y,-x)\right\|_{i, j \in\{1,2,3\}}\right], \\
& H(a, x, y)=4\left[\operatorname{det}\left\|c_{i j}(a, y,-x)\right\|_{i, j \in\{1,2\}}\right] .
\end{aligned}
$$

We construct the following $T$-comitants:

Notation 3.6.

$$
\begin{aligned}
B_{3}(a, x, y) & =\left(C_{2}, D\right)^{(1)}=\operatorname{det}\left[\operatorname{Jacobian}\left(C_{2}, D\right)\right], \\
B_{2}(a, x, y) & =\left(B_{3}, B_{3}\right)^{(2)}-6 B_{3}\left(C_{2}, D\right)^{(3)} \\
B_{1}(a) & =\operatorname{Res}_{x}\left(C_{2}, D\right) / y^{9}=-2^{-9} 3^{-8}\left(B_{2}, B_{3}\right)^{(4)} .
\end{aligned}
$$

As an example of a result using these polynomials we mention the following lemma proved in [54]: 
Lemma 3.1 (see [54]). For the existence of invariant affine straight lines in one (respectively two; three distinct) directions in the affine plane it is necessary that $B_{1}=0$ (respectively $B_{2}=0 ; B_{3}=0$ ).

Let us consider the following $G L$-comitants of systems (S):

Notation 3.7.

$$
\begin{aligned}
& M(a, x, y)=\left(C_{2}, C_{2}\right)^{(2)}=2 \operatorname{Hessian}\left(C_{2}(x, y)\right), \\
& K(a, x, y)=\operatorname{det}\left[\operatorname{Jacobian}\left(p_{2}(x, y), q_{2}(x, y)\right)\right], \\
& N(a, x, y)=K(a, x, y)+H(a, x, y), \\
& \eta(a)=\operatorname{Discrim}\left(C_{2}(x, y)\right), \\
& \mu_{0}(a)=\operatorname{Discrim}(K(a, x, y)) / 16, \\
& \theta(a)=\operatorname{Discrim}(N(a, x, y)) .
\end{aligned}
$$

Remark 3.8. We note that by the discriminant of the cubic form $C_{2}(a, x, y)$ we mean the expression given in Maple via the function "discrim $\left(C_{2}, x\right) / y^{6} "$.

The geometrical meaning of these invariant polynomials is revealed by the next three lemmas (see $[\mathbf{5 4}]$ ).

Lemma 3.2. Let $(\mathrm{S}) \in \mathrm{QS}$ and let $\mathbf{a} \in \mathbb{R}^{12}$ be its 12-tuple of coefficients. The common points of $P=0$ and $Q=0(P, Q$ are the homogenizations of $p, q$ ) on the line $Z=0$ are given by the common linear factors over $\mathbb{C}$ of $p_{2}$ and $q_{2}$. This yields the geometrical meaning of the comitants $\mu_{0}$, $K$ and $H$ :

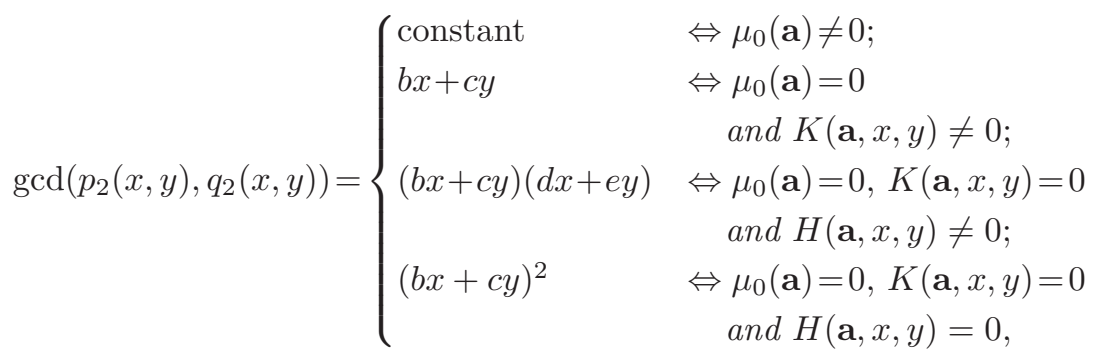

where $b x+c y, d x+e y \in \mathbb{C}[x, y]$ are some linear forms and be $-c d \neq 0$.

Lemma 3.3. A necessary condition for the existence of one couple (respectively, two couples) of parallel invariant straight lines of a system $(\mathrm{S})$ corresponding to $\mathbf{a} \in \mathbb{R}^{12}$ is the condition $\theta(\mathbf{a})=0$ (respectively, $N(\mathbf{a}, x, y)=0)$. 
Lemma 3.4. The form of the divisor $D_{\mathbb{C}}(S, Z)$ (see [55]) for systems $(\mathrm{S})$ is determined by the corresponding conditions indicated in Table 1, where we write $\omega_{1}^{c}+\omega_{2}^{c}+\omega_{3}$ if two of the points, i.e. $\omega_{1}^{c}, \omega_{2}^{c}$, are complex but not real.

Table 1

\begin{tabular}{|c|c|c|}
\hline Case & Form of $D_{\mathbb{C}}(S, Z)$ & $\begin{array}{c}\text { Necessary and sufficient } \\
\text { conditions on the comitants }\end{array}$ \\
\hline \hline 1 & $\omega_{1}+\omega_{2}+\omega_{3}$ & $\eta>0$ \\
\hline 2 & $\omega_{1}^{c}+\omega_{2}^{c}+\omega_{3}$ & $\eta<0$ \\
\hline 3 & $2 \omega_{1}+\omega_{2}$ & $\eta=0, \quad M \neq 0$ \\
\hline 4 & $3 \omega$ & $M=0, \quad C_{2} \neq 0$ \\
\hline 5 & $D_{\mathbb{C}}(S, Z)$ undefined & $C_{2}=0$ \\
\hline
\end{tabular}

Some invariant polynomials could be defined using the differential operator $\mathcal{L}=x \cdot \mathbf{L}_{2}-y \cdot \mathbf{L}_{1}$ acting on $\mathbb{R}[a, x, y]$ constructed in $[\mathbf{1 0}]$ (see also $[\mathbf{1 1}])$, where

$$
\begin{aligned}
& \mathbf{L}_{1}=2 a_{00} \frac{\partial}{\partial a_{10}}+a_{10} \frac{\partial}{\partial a_{20}}+\frac{1}{2} a_{01} \frac{\partial}{\partial a_{11}}+2 b_{00} \frac{\partial}{\partial b_{10}}+b_{10} \frac{\partial}{\partial b_{20}}+\frac{1}{2} b_{01} \frac{\partial}{\partial b_{11}}, \\
& \mathbf{L}_{2}=2 a_{00} \frac{\partial}{\partial a_{01}}+a_{01} \frac{\partial}{\partial a_{02}}+\frac{1}{2} a_{10} \frac{\partial}{\partial a_{11}}+2 b_{00} \frac{\partial}{\partial b_{01}}+b_{01} \frac{\partial}{\partial b_{02}}+\frac{1}{2} b_{10} \frac{\partial}{\partial b_{11}} .
\end{aligned}
$$

In $[\mathbf{1 0}]$ it is shown that if a polynomial $U \in \mathbb{R}[a, x, y]$ is a $G L$-comitant of system (S) then $\mathcal{L}(U)$ is also a $G L$-comitant.

So, by using this operator and the $G L$-comitant $\mu_{0}(a)=\operatorname{Res}_{x}\left(p_{2}(x, y)\right.$, $\left.q_{2}(x, y)\right) / y^{4}$ we construct the following polynomials:

$$
\begin{array}{rlrl}
\mu_{i}(a, x, y) & =\frac{1}{i !} \mathcal{L}^{(i)}\left(\mu_{0}\right), & & i=1, \ldots 4, \quad \text { where } \\
\mathcal{L}^{(i)}\left(\mu_{0}\right) & =\mathcal{L}\left(\mathcal{L}^{(i-1)}\left(\mu_{0}\right)\right), & \mathcal{L}^{0}\left(\mu_{0}\right)=\mu_{0} .
\end{array}
$$

These polynomials are in fact $G L$-comitants of systems (S). The geometrical meaning of the $G L$-comitants $\mu_{i}(a, x, y), i=0,1, \ldots, 4$ is revealed by the next two lemmas (see [55]).

Lemma 3.5. The system $P(X, Y, Z)=Q(X, Y, Z)=0$ possesses exactly four solutions counted with the multiplicities. Then $m(1 \leq m \leq 4)$ of these solutions lie on $Z=0$ if and only if for every $i \in\{0,1, \ldots, m-1\}$ we have $\mu_{i}(a, x, y)=0$ and $\mu_{m}(a, x, y) \neq 0$ as polynomials in $\mathbb{R}[x, y]$. 
Lemma 3.6. A quadratic system $(\mathrm{S})$ is degenerate (i.e. $\operatorname{gcd}(p, q) \neq$ constant) if and only if $\mu_{i}(a, x, y)=0$ as polynomials in $\mathbb{R}[x, y]$ for every $i=0,1,2,3,4$.

Using the transvectant differential operator (3.4) and the invariant polynomials $(3.2),(3.5)$ and (3.7) constructed earlier, we can define the following invariant polynomials which were used for example in [57], $[58]$ :

$$
\begin{aligned}
H_{1}(a) & \left.=-\left(\left(C_{2}, C_{2}\right)^{(2)}, C_{2}\right)^{(1)}, D\right)^{(3)}, \\
H_{2}(a, x, y) & =\left(C_{1}, 2 H-N\right)^{(1)}-2 D_{1} N, \\
H_{3}(a, x, y) & =\left(C_{2}, D\right)^{(2)}, \\
H_{4}(a) & =\left(\left(C_{2}, D\right)^{(2)},\left(C_{2}, D_{2}\right)^{(1)}\right)^{(2)}, \\
H_{5}(a) & =\left(\left(C_{2}, C_{2}\right)^{(2)},(D, D)^{(2)}\right)^{(2)}+8\left(\left(C_{2}, D\right)^{(2)},\left(D, D_{2}\right)^{(1)}\right)^{(2)}, \\
H_{6}(a, x, y) & =16 N^{2}\left(C_{2}, D\right)^{(2)}+H_{2}^{2}\left(C_{2}, C_{2}\right)^{(2)}, \\
H_{7}(a) & =\left(N, C_{1}\right)^{(2)}, \\
H_{8}(a) & =9\left(\left(C_{2}, D\right)^{(2)},\left(D, D_{2}\right)^{(1)}\right)^{(2)}+2\left[\left(C_{2}, D\right)^{(3)}\right]^{2}, \\
H_{9}(a) & =-\left(\left((D, D)^{(2)}, D\right)^{(1)}, D\right)^{(3)}, \\
H_{10}(a) & =\left((N, D)^{(2)}, D_{2}\right)^{(1)}, \\
H_{11}(a, x, y) & =8 H\left[\left(C_{2}, D\right)^{(2)}+8\left(D, D_{2}\right)^{(1)}\right]+3 H_{2}^{2}, \\
H_{12}(a, x, y) & =(D, D)^{(2)} \equiv \mathrm{Hessian}^{(D)}, \\
N_{1}(a, x, y) & =C_{1}\left(C_{2}, C_{2}\right)^{(2)}-2 C_{2}\left(C_{1}, C_{2}\right)^{(2)}, \\
N_{2}(a, x, y) & =D_{1}\left(C_{1}, C_{2}\right)^{(2)}-\left(\left(C_{2}, C_{2}\right)^{(2)}, C_{0}\right)^{(1)}, \\
N_{5}(a, x, y) & =\left[\left(D_{2}, C_{1}\right)^{(1)}+D_{1} D_{2}\right]^{2}-4\left(C_{2}, C_{2}\right)^{(2)}\left(C_{0}, D_{2}\right)^{(1)}, \\
\mathcal{G}_{1}(a) & =\left(\left(C_{2}, \widetilde{E}\right)^{(2)}, D_{2}\right)^{(1)}, \\
\mathcal{G}_{3}(a) & =8 H_{8}-9 H_{5}, \\
& \left(\mu_{0}-\eta\right)_{1}-6 \eta\left(H_{4}+12 H_{10}\right),
\end{aligned}
$$

where in the formula for $\mathcal{G}_{1}, \widetilde{E}=\left[D_{1}\left(2 \omega_{1}-\omega_{2}\right)-3\left(C_{1}, \omega_{1}\right)^{(1)}-D_{2}\left(3 \omega_{3}+\right.\right.$ $\left.\left.D_{1} D_{2}\right)\right] / 72, \omega_{1}=\left(C_{2}, D_{2}\right)^{(1)}, \omega_{2}=\left(C_{2}, C_{2}\right)^{(2)}$ and $\omega_{3}=\left(C_{1}, D_{2}\right)^{(1)}$.

3.6. Interaction between topological and polynomial invariants. On polynomial vector fields, we have the topological equivalence relation 
and also the affine equivalence relation. We also have the equivalence relation induced by the larger group $\operatorname{Aff}(2, \mathbb{R}) \times R^{*}$ of affine transformations and time rescaling.

We say that two planar polynomial vector fields are equivalent via the group $\operatorname{Aff}(2, \mathbb{R}) \times R^{*}$ if and only if there exists an affine transformation and a time homothety transforming the first vector field into the second by this transformation.

Both the second and third equivalence relations are strictly finer than the topological one. Indeed, for example if two systems are affinely equivalent then clearly they are also topologically equivalent but not necessarily viceversa. So each topological equivalence class splits into several (usually an infinite number of) equivalence classes by this affine group action. However, assuming we list all equivalence classes via this group action, then we could also get by further identification the topological equivalence classes.

The topological invariants are difficult and even often impossible to calculate. However, the polynomial invariants under the group action could easily be computed using symbolic computations. This yields a powerful tool for the qualitative study of polynomial vector fields, a thing which was observed by C. S. Sibirsky, the founder of the algebraic invariant theory of polynomial vector fields. The polynomial invariants were sufficient for the classification and even for the determination of the bifurcation diagram in the 12-dimensional space of coefficients of the systems for quite a number of families of quadratic vector fields as we previously indicated.

Once a minimal list of phase portraits is found for a family of polynomial vector fields, a natural question to ask is:

What are the necessary and sufficient conditions for the realization of each one of the phase portraits in this list?

For a number of families of polynomial vector fields this problem was completely solved by using polynomial invariants (see for example [56], $[\mathbf{5 7}],[\mathbf{5 9}],[\mathbf{6 2}])$. For these as well as for other families, the polynomial invariants were sufficient to obtain the topological classification. In general, the polynomial invariants need however to be used in conjunction with other methods, analytical, geometric and numerical, to obtain full results (see for example [5] ).

3.7. Normal forms and bifurcation diagrams. Another problem is to find out how phase portraits change when we move in the parameter 
space. The drawing of the bifurcation diagram gives, as we shall see below, only a partial answer to this question. We recall that a bifurcation diagram is essentially the splitting of the parameter space into distinct parts, separated by bifurcation strata formed by points of bifurcation, i.e. points in the neighborhood of which there are at least two distinct phase portraits.

If a normal form for a family depends on $m$ parameters, then the bifurcation diagram can be drawn in $\mathbb{R}^{m}$. But not always the parameter space is $\mathbb{R}^{m}$ or one of its subspaces. Sometimes it is a manifold requiring several charts or even a more complicated space.

Let us consider for example the family $\mathbf{Q C}$ of quadratic systems with a center. Placing the center at the origin, any such system can be brought by affine transformations and time rescaling to the Kapteyn-Bautin normal form (see for example $[\mathbf{1 2}]$ ):

$$
d x / d t=-y-b x^{2}-C x y-d y^{2}, \quad d y / d t=x+a x^{2}+A x y-a y^{2} .
$$

This normal form depends on 5 parameters but because we can also rescale $(x, y) \mapsto(\lambda x, \lambda y), \lambda \in \mathbb{R} \backslash\{0\}$ the parameter space is here a 4-dimensional manifold, the projective space $\mathbb{P R}^{4}$, where we need to work with 5 charts. Each system in the union of the family QWF of quadratic systems possessing a weak focus with the family QC can be brought to this normal form by first placing the weak focus or the center at the origin and then acting with the group.

It turns out that the family $\mathbf{Q C}$ is the union of the following families of systems (see $[\mathbf{5 1}])$ :

The class SymC of symmetric systems with a center; the family LV-C of the so called Lotka-Volterra systems with center defined further below; the class Ham of the Hamiltonian systems with center and the family Cod 4 of the codimension 4 systems with center, which are characterized by having two invariant algebraic curves: an irreducible singular cubic curve and a conic, more precisely a parabola whose point at infinity is the singular point of the cubic (see [51]).

In the parameter space $\mathbb{P R}^{4}$ considered above, the systems with a center form an algebraic set which splits into four irreducible components defined by the following sets of conditions which correspond to SymC, L-V-C, Ham, Cod4: $a=0=C$ for systems in SymC; $a=0=b+d=$ 0 , the class of systems which can be brought to this canonical form via an affine transformation and time rescaling is called in the literature the Lotka-Volterra class of systems with a center, denoted here by $\mathbf{L}-\mathbf{V}-\mathbf{C}$; $C+2 a=0=A-2 b$ for systems in Ham; $C+2 a=0=A+3 b+5 d=$ $a^{2}+b d+2 d^{2}$ for systems in $\operatorname{Cod} 4$. 
The first three families are planes in $\mathbb{P R}^{4}$ and the last family is a conic situated in the plane $C+2 a=0=A+3 b+5 d$ of $\mathbb{P R}^{4}$. In each one of the planes only three parameters are involved: for systems in SymC these are $b, A, d$; for those in Ham they are $a, b, A$ and for those in L-V-C they are $C, A, b$.

In the author's view to call the systems in the family $\mathbf{L}-\mathbf{V}-\mathbf{C}$ the Lotka-Volterra systems with center is to use a misnomer. This terminology, was introduced in $[\mathbf{7 6}]$ for the family of systems which using our coordinates is defined by the equations $a=0$ and $b+d=0$. In general in the literature, the term Lotka-Volterra was used for quadratic systems which have at least two real invariant lines. Taking the affine part of the projective plane defined by the equations $a=0$ and $b+d=0$, by taking $d=1$ and hence $b=-1$ and letting the remaining parameters satisfy $C^{2}+4 b(A+b)<0$ (this clearly defines an open set in this affine plane), we have systems which have only one real invariant line, i.e. $y-1 / A=0$ for $A \neq 0$. They also have two complex, non-real invariant lines which are given by the equations: $\left(C \pm \sqrt{C^{2}+4 b(A+b)}\right) x / 2-b y+1=0$.

So an open set in $\mathbb{P R}^{2}$ defined by $a=0=b+d$ are not Lotka-Volterra systems since they do not have two real invariant lines. In fact out of the 7 phase portraits of the so-called Lotka-Volterra quadratic systems with a center only 3 are Lotka-Volterra.

Although it would be natural to denote by $\mathbf{L}-\mathbf{V}-\mathbf{C}$ the class of all Lotka-Volterra systems (i.e. systems which have at least two real invariant lines) which have a center, we do not do this here, not to conflict with the terminology in the literature and in particular with $[\mathbf{7 6}]$. We stress that, as already indicated above, in this work we use the notation L-V-C to denote the family of systems which can be reduced by affine transformations and time rescaling to the Kapteyn-Bautin normal form with $a=0=b+d$, analogously to the terminology in [76].

To visualize the bifurcation diagram of $\mathbf{Q C}$ we work with each case separately (see [51]). In each one of the cases SymC, L-V-C, Ham, using the above normal form, we get a parameter space which is the real projective plane $\mathbb{P R}^{2}$. These bifurcation diagrams can be seen in $[\mathbf{5 1}]$. These building blocks of the bifurcation diagram of $\mathbf{Q C}$ are glued in $\mathbb{P R}^{4}$ along the intersection of these four strata. For example SymC and LV-C are glued together along the line contained in the hyperplane $a=0$ defined by the equations $b+d=0$ and $C=0$. We obtain in this way the bifurcation diagram of this family within the space $\mathbb{P R}^{4}$. But this bifurcation diagram does not accurately convey to us some of the important phenomena occurring in $\mathbf{Q C}$ as we explain below. 
Consider the system $S: d x / d t=-y-x^{2}, d y / d t=x-x y$ belonging to the family SymC and for which the parameters in $\mathbb{P R}^{4}$ are $A=-1$, $b=1$ and $C=0=a=d$. For this system we thus have $b+d=1 \neq 0$ and hence $S$ does not belong to the projective plane $a=0=b+d$ of the family L-V-C in our parameter space $\mathbb{P R}^{4}$. Also $S$ cannot be perturbed within $\mathbb{P R}^{4}$ so as to get a system in the plane $a=0=b+d$. Indeed the only systems in the plane $a=0=C$ which can be perturbed within the plane $a=0=b+d$ are those of the common line of the two planes and $S$ does not belong to this line. Thus no sequence of elements of L-V-C contained in our parameter space could have as a limit point the system $S$. However, as we shall later see, $S$ is a limit point of $\mathbf{L}-\mathbf{V}$ C. The reason for this is that the systems in $\mathbf{L}-\mathbf{V}-\mathbf{C}$ contained in our parameter space are only representatives of their corresponding affine equivalence classes forced by our choosing the specific normal form of the systems above. But as we shall later see, moving on affine orbits of L-V-C we can obtain as a limit point of $\mathbf{L}-\mathbf{V}-\mathbf{C}$ the system $S$.

Other properties of $S$ are: i) the origin is a center for $S$, ii) all points at infinity of $S$ are singular points and this is the only quadratic system with a center in our parameter space with this property, making this system (as well as its affine equivalents) the most degenerate one of the systems with center, iii) $S$ has the invariant line $y-1=0$ and two more affine invariant complex lines $x \pm i y=0$. Due to ii) and iii) the system is Darboux integrable and it has a rational quadratic first integral of degree two. Due to the properties ii) and iii) we can apply to this system the results on the moduli spaces constructed in [59] and obtain the following result.

Theorem 3.2. Any symmetric system with center and with all points at infinity as singularities is a limit point of the space of $\boldsymbol{L}-\boldsymbol{V}-\boldsymbol{C}$ systems.

Proof: Consider again the system $S$ defined above. We observe that $S$ can be obtained from the following normal form $\left(C_{2} .2\right)$ occurring in [59]:

$$
d x / d t=(x+\delta)^{2}+1+y, \quad d y / d t=x y,
$$

where $\delta \in \mathbb{R}$ in the following way.

Consider the system $S^{\prime}$ in this form for which $\delta=0$, i.e. the system $d x / d t=1+y+x^{2}, d y / d t=x y$ with phase portrait Picture $C_{2} \cdot 2(b)$ below which was constructed in $[\mathbf{5 9}$, p. 755$]$. We give here the full picture in $[\mathbf{5 9}]$ which shows that the systems with the configuration of invariant lines Config. $C_{2} .2$ (having one real and two (dotted) complex invariant lines) 
correspond to two topologically distinct phase portraits, one of them being Picture $C_{2} \cdot 2(b)$, the phase portrait of $S$ and of $S^{\prime}$. Translating the point $(0,-1)$ at the origin, after denoting the new coordinates also by $x, y$, the equations become: $d x / d t=y+x^{2}, d y / d t=-x+x y$. By rescaling the time $T=-t$ we obtain our previous system $S$.

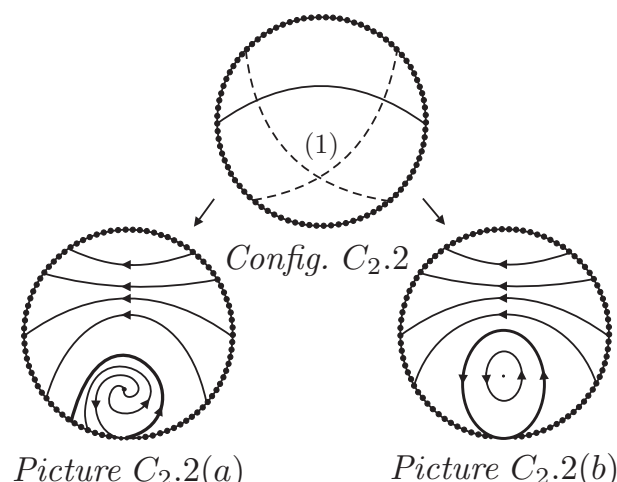

We now consider perturbations $\left(C_{2 \varepsilon, \delta} .2\right)$ of the systems in the above normal form found in $[\mathbf{5 9}$, p. 771]:

$$
\begin{aligned}
& d x / d t=(x+\delta)^{2}+1+y-\left(\varepsilon / \varphi^{+}\right) y^{2}, \\
& d y / d t=(1+\varepsilon) x y+\left(2 \delta \varepsilon(1+\varepsilon) / \varphi^{+}\right) y^{2},
\end{aligned}
$$

where $\varphi^{+}(\delta, \varepsilon)=\delta^{2}(\varepsilon+1)^{2}+(\varepsilon-1)^{2}$ which for $\varepsilon$ tending to zero has the limit $\varphi^{+}(\delta, 0)=\delta^{2}+1 \neq 0$.

All the systems in $\left(C_{2 \varepsilon, \delta} .2\right)$ have the following affine invariant lines: $y=0, y+[\varepsilon(\delta \pm i)+\delta \mp i](x+\delta \pm i)=0$ in addition to the line at infinity. Having invariant lines of total multiplicity four we can apply to this family results contained in $[\mathbf{5 9}]$ and $[\mathbf{5 8}]$, in particular we can use the bifurcation diagram in the twelve parameter space $\mathbb{R}^{12}$ of coefficients, which appears on [58, p. 54]. The configuration in which we are interested here is Config. 4.2, i.e. the configuration of one real and two complex (not real) affine invariant lines, plus the invariant line at infinity. To determine the phase portraits for this configuration we need the values of the following polynomial invariants: $\eta, B_{3}, \theta, H_{7}, \mu_{0}, \mathcal{G}_{1}$ which appear in Table 2 of [58] and use this table.

Calculating these invariants for the systems in the perturbation we obtain: $\left.\eta=-4 \varepsilon^{4}(\varepsilon-1)^{2}\right) /\left(\varphi^{+}\right)^{2}$ so we have $\eta<0 ; B_{3}=0 ; \theta=$ 
$8 \varepsilon\left(1-\varepsilon^{2}\right)(1+\varepsilon) / \varphi^{+}$so we have $\theta \neq 0 . \mu_{0}=-\varepsilon\left(1-\varepsilon^{2}\right)^{2}\left(1+\delta^{2}\right) /\left(\varphi^{+}\right)^{2}$, $H_{7}=4\left(1-\varepsilon^{2}\right)$ and $\mathcal{G}_{1}=2 \delta \varepsilon^{2}(1-\varepsilon)^{2}(1+\varepsilon)\left[\delta^{2}(1+\varepsilon)^{2}+(\varepsilon+3)^{2}\right]$.

As an illustration of how the bifurcation diagram in the twelve-dimensional space of coefficients can be used, we determine the phase portraits of the systems in the perturbation, by applying here the invariants we used in [58] and mentioned above.

We see in Table 2 of [58] that the phase portrait Picture $4.2(c)$ is a phase portrait of $\mathbf{L}-\mathbf{V}-\mathbf{C}$ systems which in our parameter space corresponds to the condition $C^{2}+4 b(A+b)<0$ (see $\left.[\mathbf{5 1}]\right)$, a generic region in this parameter space $\mathbb{P R}^{4}$. When we take $\delta=0$, the family above becomes a perturbation depending on a small number $\varepsilon>0$ of the system in $\left(C_{2 \varepsilon, \delta} .2\right)$

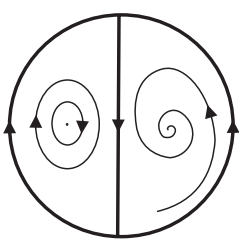

Picture $4.2(c)$ with $\delta=0=\varepsilon$. According to the Table 2 in $[\mathbf{5 8}]$ to obtain the portrait $4.2(\mathrm{c})$ we need to have $\eta<0, B_{3}=0, \theta \neq 0, H_{7} \neq 0, \mu_{0}<0$, $\mathcal{G}_{1} \neq 0$. Or clearly $\varphi^{+}>0$ and we can easily see from the formulas above that for $\delta \neq 0$ and $\epsilon>0$, all these conditions are satisfied. So the symmetric quadratic system with center and with the line at infinity filled up with singularities is a limit point of systems in the class $\mathbf{L}-\mathbf{V}-\mathbf{C}$ of the so called Lotka-Volterra systems with a center.

The above result could not be detected from our bifurcation diagram in $\mathbb{P R}^{4}$ because this diagram only contains representatives of equivalence relations via the groups actions.

Observation. The subset of $\mathbb{P R}^{4}$ obtained by gluing these four components is not yet a minimal set of representatives of all quadratic systems with a center via the affine group action. Indeed, we could easily convince ourselves that the second case ( $\mathbf{L}-\mathbf{V}-\mathbf{C})$ has the symmetry $(x, y) \rightarrow(-x,-y)$ so that to obtain a set of representatives of $\mathbf{L}-\mathbf{V}-\mathbf{C}$ we need to make this identification. We could visualize this space as half a disk with the opposite points on the diameter identified. A minimal set of representatives of each class of systems with a center modulo the group action can be obtained from $\mathbb{P R}^{4}$ after making such identifications.

The literature contains many bifurcation diagrams for families of quadratic systems. They are usually done with respect to the parameters of the corresponding normal forms. These are, up to a point, helpful for visualizing how systems change when we change the values of the parameters. But as indicated by the result above, such bifurcation diagrams do not convey to us all the interesting phenomena involved. To see these 
phenomena we would need to construct moduli spaces as described in the next section.

For the whole family QS of quadratic systems the parameter space is $\mathbb{R}^{12}$, the space of the twelve coefficients of the systems. Usually, for specific families, one works with normal forms which may involve a smaller number of coefficients. The bifurcation diagram is then given in terms of these smaller number of parameters. However, since the same systems are sometimes considered with respect to other normal forms, it is best to also be able to see how phase portraits change when we consider them belonging to $\mathbb{R}^{12}$ which contains the systems of all normal forms.

The invariant polynomials can give us, for certain families, their bifurcation diagrams in the full 12-parameter space of coefficients, so no matter in which normal form the systems are presented we could see what are its neighboring points and which are the bifurcation points of the family. For example see the bifurcation diagrams in the 12-parameter space in $[\mathbf{6 2}],[\mathbf{5 7}],[\mathbf{5 6}],[\mathbf{5 9}]$.

If a family is more complex, then polynomial invariants help in the first part of the construction of the bifurcation diagram. For example in the case of the whole family QS we can completely describe the global behavior of the configurations of singularities at infinity in terms of polynomial invariants (see $[6]$ ). For understanding other features of the family QS we would need to combine the use of polynomial invariants with other methods such as for example geometric methods or methods of numerical analysis (see [5]).

3.8. Moduli spaces versus parameter spaces. We cannot end this article without mentioning here moduli spaces. The notion of moduli space goes back to Riemann who showed that compact Riemann surfaces of genus $g$ depend on $3 g-3$ complex parameters which Riemann called moduli.

Moduli spaces are encountered in geometric classification problems. As Ben-Zvi says in [13] "Moduli spaces can be viewed as geometric solutions to geometric classification problems". We have a set $X$ of geometric objects which is endowed with an equivalence relation $\sim$ and we would like to see how the objects vary or modulate when they move in $X$. We can form the quotient set $X / \sim$ and usually one tries to endow this quotient set with a structure, for example a topological or an analytic structure or the structure of an algebraic variety.

Apart from being analytic and algebraic objects, planar polynomial vector fields are also geometric objects. Since we are interested in classifying families of such vector fields we should be interested in moduli 
spaces which would give us geometric solutions to these geometric classification problems.

Moduli spaces are abundant in algebraic geometry, differential geometry and algebraic topology and they are currently much studied. They could be of great help. For example a moduli space (see [34]) intervened in an essential way in the proof given by S. Donaldson of the theorem that on four-dimensional topological manifolds we could have distinct smooth structures.

Example 6. One of the simplest moduli spaces is the set of all 1dimensional subspaces of $\mathbb{R}^{2}$. These are the lines through the origin. The set of all these lines could be given the structure of a 1-dimensional real analytic manifold, the real projective line $\mathbb{P R}$. Due to this we know what it means to move a line analytically in this real analytic manifold. An analytic family of lines through the origin in $\mathbb{R}^{2}$ can thus be viewed as an analytic map $M \rightarrow \mathbb{P R}$ from a real analytic manifold $M$ into $\mathbb{P}$. We say that $\mathbb{P R}$ is the moduli space of analytically varying families of lines in $\mathbb{R}^{2}$, passing through the origin.

Analogously we can construct the moduli space of the set of all the 1dimensional subspaces of $\mathbb{R}^{n+1}$ obtaining the real analytic manifold $\mathbb{P}^{n}$.

Example 7. This example is described in detail in the article $[\mathbf{1 3}]$ of the book [28]. See also $[\mathbf{3 0}]$. Let $X$ be the set of all compact Riemann surfaces of genus 1 with the biholomorphism equivalence relation, i.e. two Riemann surfaces $T_{1}, T_{2}$ of genus 1 are equivalent if and only if there exists a bijective holomorphic map $T_{1} \rightarrow T_{2}$ which has an holomorphic inverse. It turns out that any such Riemann surface can be obtained in the following way:

Consider a lattice $L$, given by two complex numbers $\omega_{1}, \omega_{2}$ which are linearly independent over the reals. Hence the quotient $\omega_{2} / \omega_{1}$ is not a real number. The numbers $\omega_{1}, \omega_{2}$ generate the subgroup $L=$ $\left\{n_{1} \omega_{1}+n_{2} \omega_{2} \mid n_{1}, n_{2} \in \mathbb{Z}\right\}$ of $\mathbb{C}$ and we can form the quotient group $\mathbb{C} / L$ with neutral element $e$ which is the equivalence class of $0 \in \mathbb{C}$. We can endow the quotient group $\mathbb{C} / L$ with the complex analytic structure inherited from $\mathbb{C}$ via the canonical map $\mathbb{C} \rightarrow \mathbb{C} / L$. We obtain in this way a compact Riemann surface $T=\mathbb{C} / L$ of genus 1 which is topologically the torus $S^{1} \times S^{1}$. Two lattices $L$ and $\lambda L$ where $\lambda \in \mathbb{C} \backslash\{0\}$ yield holomorphically equivalent Riemann surfaces. Therefore the set of lattices modulo multiplication by a non-zero complex number parametrize the genus 1 Riemann surfaces. Let us now assume that $\omega_{1}, \omega_{2}$ is a positively 
oriented basis on $L$ over $\mathbb{Z}$. Since $n_{1} \omega_{1}+n_{2} \omega_{2}=\omega_{1}\left(n_{1}+n_{2} \omega\right)$ and since lattices $L$ and $\left(\omega_{1}\right)^{-1} L$ yield holomorphic Riemann surfaces, we may assume that our lattice is defined by the complex numbers $1, \omega$ over $\mathbb{Z}$ and we get $\omega$ in the upper half plane $\mathbb{H}$ and any $\omega$ in $\mathbb{H}$ determines a unique such oriented lattice $L=\mathbb{Z}+\omega \mathbb{Z}$.

Two such Riemann surfaces $T_{1}$ and $T_{2}$ defined by lattices $L, L^{*}$ are holomorphically equivalent if and only if $\omega=\omega_{2} / \omega_{1}, \omega^{*}=\omega_{2}^{*} / \omega_{1}^{*}$ are related by $\omega^{*}=\left(a_{11} \omega+a_{12}\right) /\left(a_{21} \omega+a_{22}\right)$, for $a_{11}, \ldots, a_{22} \in \mathbb{Z}$. Thus the set of all complex structures on a torus correspond to all complex numbers $\omega \in \mathbb{H}$ modulo the action of the group of transformations $\omega \rightarrow \omega *$ indicated before. This resulting space could be considered as the moduli space of the compact Riemann surfaces of genus 1 .

In general one wants to endow the quotient set $X / \sim$ with a geometric structure depending on the problem at hand, as we illustrated in the above examples. The set $X / \sim$ endowed with this geometric structure will be the moduli space associated to the classification problem.

A moduli space of polynomial vector fields with respect to the action of the group of affine transformations and time rescaling were considered by the author together with Vulpe in [59], where the vector fields were quadratic and with the line at infinity filled up with singularities. It was in this work that we noticed that the system $S$ in the preceding section could be perturbed to give us systems in $\mathbf{L}-\mathbf{V}-\mathbf{C}$, phenomenon that could only be seen on the moduli space and motivates us to study such spaces. In [60] moduli spaces were constructed for some families of quadratic vector fields possessing invariant lines with total multiplicity four and having three real singularities at infinity.

As a subspace of $\mathbb{R}^{12}$, QC inherits a topological structure. Let $\sim$ be the affine equivalence relation. With the help of the canonical map $\mathrm{QC} \rightarrow \mathrm{QC} / \sim$ this structure induces a topology on $\mathbf{Q C} / \sim$ where we can talk of continuous families of elements. But it is not so easy to understand the space $\mathbf{Q C} / \sim$. To understand it, we need to associate to each element of $\mathbf{Q C}$ an object in an analogous manner to the way we associated in Example 7, to each genus 1 compact Riemann surface, the equivalence class of $[\omega]$ in the upper half-plane modulo the group action. In both cases $[\mathbf{5 9}]$ and $[\mathbf{6 0}]$, where we constructed moduli spaces, we had families of systems with invariant lines. All systems QC have invariant curves. Systems in (L-V-C) have invariant lines, others systems in QC have invariant conics or invariant irreducible cubic curves. This gives us the hint of using these invariant curves occuring in systems in QC for the purpose of giving structure to $\mathrm{QC} / \sim$. 
Acknowledgements. The inspiration to write this article came from my recent joint work with $\mathrm{N}$. Vulpe, who taught me many things about polynomial invariants, and also from reading splendid chapters, in particular [65] and [13] in the book [28], edited by Timothy Gowers et al. Many thanks are due to J. Llibre, N. Vulpe and J. C. Artés for help in making the list of references as extensive as we could, and for providing valuable information contained in their work in these references.

Thanks are also due to the two referees for indicating typographical errors and for minor corrections.

\section{References}

[1] J. C. Artés, R. E. KooiJ, And J. Llibre, Structurally stable quadratic vector fields, Mem. Amer. Math. Soc. 134(639) (1998), 108 pp.

[2] J. C. Artés And J. Llibre, Quadratic Hamiltonian vector fields, J. Differential Equations 107(1) (1994), 80-95. DOI: 10.1006/jdeq. 1994.1004.

[3] J. C. Artés And J. Llibre, Phase portraits for quadratic systems having a focus and one antisaddle, Rocky Mountain J. Math. 24(3) (1994), 875-889. DOI : 10.1216/rmjm/1181072378.

[4] J. C. Artés And J. Llibre, Corrigendum: A correction to the paper "Quadratic Hamiltonian vector fields" [J. Differential Equations 107(1) (1994), 80-95], J. Differential Equations 129(2) (1996), 559-560. DOI: 10.1006/jdeq.1996.0127.

[5] J. C. Artés, J. Llibre, And D. Schlomiuk, The geometry of quadratic differential systems with a weak focus of second order, Internat. J. Bifur. Chaos Appl. Sci. Engrg. 16(11) (2006), 3127-3194. DOI: $10.1142 / \mathrm{S} 0218127406016720$.

[6] J. C. Artés, J. Llibre, And D. Schlomiuk, From topological to geometric equivalence in the classification of singularities at infinity for quadratic vector fields, Rocky Mountain J. Math. (to appear).

[7] J. C. Artés, J. Llibre, And N. Vulpe, Singular points of quadratic systems: a complete classification in the coefficient space $\mathbb{R}^{12}$, Internat. J. Bifur. Chaos Appl. Sci. Engrg. 18(2) (2008), 313-362. DOI : $10.1142 / \mathrm{S} 021812740802032 X$.

[8] J. C. Artés, J. Llibre, And N. Vulpe, Quadratic systems with an integrable saddle: a complete classification in the coefficient space $\mathbb{R}^{12}$, Nonlinear Anal. 75(14) (2012), 5416-5447. DOI: $10.1016 / j . n a .2012 .04 .043$. 
[9] J. C. Artés, J. Llibre, and N. Vulpe, Complete geometric invariant study of two classes of quadratic systems, Electron. J. Differential Equations 2012(9) (2012), 1-35.

[10] V. A. Baltag And N. I. VulPe, Total multiplicity of all finite critical points of the polynomial differential system, Planar nonlinear dynamical systems (Delft, 1995), Differential Equations Dynam. Systems 5(3-4) (1997), 455-471.

[11] V. A. Baltag and N. I. Vulpe, The differential operators and multiplicity of singular points for polynomial differential system, Bul. Acad. Ştiinţe Repub. Mold. Mat. 1998(1) (1998), 81-94, 151, 154.

[12] N. N. Bautin, On the number of limit cycles appearing with variation of the coefficients from an equilibrium state of the type of a focus or a center, (Russian), Mat. Sbornik N.S. 30(72) (1952), 181-196; translation in: American Math. Soc. Translation 100 (1954), 19 pp.

[13] D. D. Ben-Zvi, Moduli spaces, in: "The Princeton Companion to Mathematics" (T. Gowers, J. Barrow-Green, I. Leader, eds.), Princeton University Press, Princeton, NJ, 2008, pp. 408-419.

[14] C. A. Buzzi, J. Llibre, and J. C. R. Medrado, Phase portraits of reversible linear differential systems with cubic homogeneous polynomial nonlinearities having a non-degenerate center at the origin, Qual. Theory Dyn. Syst. 7(2) (2009), 369-403. DOI: $10.1007 / \mathrm{s} 12346-008-0021-2$.

[15] L. CAIRÓ AND J. LliBRE, Phase portraits of quadratic polynomial vector fields having a rational first integral of degree 2, Nonlinear Anal. 67(2) (2007), 327-348. DOI: 10.1016/j.na.2006.04.021.

[16] L. CAIRÓ AND J. Llibre, Phase portraits of cubic polynomial vector fields of Lotka-Volterra type having a rational first integral of degree 2, J. Phys. A 40(24) (2007), 6329-6348. DOI : 10.1088/1751$8113 / 40 / 24 / 0051$.

[17] F. CAO AND J. JiAng, The classification on the global phase portraits of two-dimensional Lotka-Volterra system, J. Dynam. Differential Equations 20(4) (2008), 797-830. DOI: 10.1007/s10884-0089122-5.

[18] M. Carbonell and J. Llibre, Chordal cubic systems, Publ. Mat. 33(2) (1989), 253-311. DOI: 10.5565/PUBLMAT_33289_07.

[19] M. Caubergh, J. Llibre, and J. Torregrosa, Global classification of a class of cubic vector fields whose canonical regions are period annuli, Internat. J. Bifur. Chaos Appl. Sci. Engrg. 21(7) (2011), 1831-1867. DOI: 10.1142/S02181274110295017. 
[20] M. Caubergh, J. Llibre, and J. Torregrosa, Global phase portraits of some reversible cubic centers with collinear or infinitely many singularities, Internat. J. Bifur. Chaos Appl. Sci. Engrg. 22(11) (2012), 20 p. DOI: 10.1142/S0218127412502732.

[21] A. Cima And J. Llibre, Algebraic and topological classification of the homogeneous cubic vector fields in the plane, J. Math. Anal. Appl. 147(2) (1990), 420-448. DOI: 10.1016/0022-247X (90) 90359-N.

[22] B. Coll, A. Ferragut, and J. Llibre, Phase portraits of the quadratic systems with a polynomial inverse integrating factor, Internat. J. Bifur. Chaos Appl. Sci. Engrg. 19(3) (2009), 765-783. DOI : $10.1142 / \mathrm{S} 0218127409023299$.

[23] B. Coll, A. Gasull, And J. Llibre, Some theorems on the existence, uniqueness, and nonexistence of limit cycles for quadratic systems, J. Differential Equations 67(3) (1987), 372-399. DOI : $10.1016 / 0022-0396$ (87)90133-1.

[24] B. Coll, A. Gasull, And J. Llibre, Quadratic systems with a unique finite rest point, Publ. Mat. 32(2) (1988), 199-259. DOI : $10.5565 /$ PUBLMAT_ $_{-} 32288_{-} 08$.

[25] B. García, J. Llibre, and J. S. PÉrez Del Río, Phase portraits of the quadratic vector fields with a polynomial first integral, Rend. Circ. Mat. Palermo (2) 55(3) (2006), 420-440. DOI : $10.1007 /$ BF02874780.

[26] A. Gasull, S. Li-Ren, And J. Llibre, Chordal quadratic systems, Rocky Mountain J. Math. 16(4) (1986), 751-782. DOI: 10. 1216/RMJ-1986-16-4-751.

[27] A. Gasull and R. Prohens, Quadratic and cubic systems with degenerate infinity, J. Math. Anal. Appl. 198(1) (1996), 25-34. DOI: $10.1006 /$ jmaa.1996.0065.

[28] T. Gowers, J. Barrow-Green, and I. Leader, Eds., "The Princeton Companion to Mathematics", Princeton University Press, Princeton, NJ, 2008.

[29] J. H. GRACE AND A. Young, "The algebra of invariants", Reprint of the 1903 original, Cambridge Library Collection, Cambridge University Press, Cambridge, 2010. DOI : 10.1017/CB09780511708534.

[30] R. C. Gunning, "Lectures on Riemann surfaces", Princeton Mathematical Notes, Princeton University Press, Princeton, N.J., 1966.

[31] P. DE JAGER, Phase portraits for quadratic systems with a higher order singularity with two zero eigenvalues, J. Differential Equations 87(1) (1990), 169-204. DOI: 10.1016/0022-0396(90)90021-G. 
[32] X. Jarque, J. Llibre, and D. S. Shafer, Structurally stable quadratic foliations, Rocky Mountain J. Math. 38(2) (2008), 489-530. DOI : 10.1216/RMJ-2008-38-2-489.

[33] Yu. F. Kalin and N. I. Vulpe, Affine-invariant conditions for the topological discrimination of quadratic Hamiltonian differential systems, (Russian), Differ. Uravn. 34(3) (1998), 298-302, 428; translation in: Differential Equations 34(3) (1998), 297-301.

[34] D. Kotschick, Gauge theory is dead!-Long live gauge theory!, Notices Amer. Math. Soc. 42(3) (1995), 335-338.

[35] C. Z. Li, J. Llibre, And Z. F. Zhang, Weak focus, limit cycles, and bifurcations for bounded quadratic systems, J. Differential Equations 115(1) (1995), 193-223. DOI: 10.1006/jdeq.1995.1012.

[36] J. Llibre, A. MAhdi, And N. Vulpe, Phase portraits and invariant straight lines of cubic polynomial vector fields having a quadratic rational first integral, Rocky Mountain J. Math. 41(5) (2011), 1585-1629. DOI : 10.1216/RMJ-2011-41-5-1585.

[37] J. Llibre And J. C. Medrado, Darboux integrability and reversible quadratic vector fields, Rocky Mountain J. Math. 35(6) (2005), 1999-2057. DOI : 10.1216/rmjm/1181069627.

[38] J. Llibre And R. D. S. Oliveira, Phase portraits of quadratic polynomial vector fields having a rational first integral of degree 3 , Nonlinear Anal. 70(10) (2009), 3549-3560. DOI: 10.1016/j.na. 2008.07.012.

[39] J. Llibre And R. D. S. Oliveira, Erratum to "Phase portraits of quadratic polynomial vector fields having a rational first integral of degree 3" [Nonlinear Anal. 70(10) (2009), 3549-3560], Nonlinear Anal. 71(12) (2009), 6378-6379. DOI: 10.1016/j.na.2009.06.002.

[40] J. Llibre AND D. Schlomiuk, The geometry of quadratic differential systems with a weak focus of third order, Canad. J. Math. 56(2) (2004), 310-343. DOI: 10.4153/CJM-2004-015-2.

[41] J. Llibre And X. Zhang, Topological phase portraits of planar semi-linear quadratic vector fields, Houston J. Math. 27(2) (2001), 247-296.

[42] M. Lupan AND N. Vulpe, Classification of quadratic systems with a symmetry center and simple infinite singular points, In memory of C. S. Sibirsky, Bul. Acad. Ştiinţe Repub. Mold. Mat. 2003(1) (2003), 102-119.

[43] L. MArkus, Global structure of ordinary differential equations in the plane, Trans. Amer. Math. Soc. 76 (1954), 127-148. DOI: 10. 1090/S0002-9947-1954-0060657-0. 
[44] I. V. Nikolaev and N. I. Vulpe, Topological classification of quadratic systems with a unique finite second order singularity with two zero eigenvalues, Izv. Akad. Nauk Respub. Moldova Mat. 1993(1) (1993), 3-8, 107, 109.

[45] I. V. Nikolaev and N. I. Vulpe, Topological classification of quadratic systems at infinity, J. London Math. Soc. (2) 55(3) (1997), 473-488. DOI: 10.1112/S0024610796004735.

[46] P. J. Olver, "Classical invariant theory", London Mathematical Society Student Texts 44, Cambridge University Press, Cambridge, 1999. DOI: 10.1017/CB09780511623660.

[47] J. PAL And D. Schlomiuk, Summing up the dynamics of quadratic Hamiltonian systems with a center, Canad. J. Math. 49(3) (1997), 583-599. DOI : 10.4153/CJM-1997-027-0.

[48] J. W. REYN, Phase portraits of a quadratic system of differential equations occurring frequently in applications, Nieuw Arch. Wisk. (4) 5(2) (1987), 107-155.

[49] C. Rousseau and D. Schlomiuk, Cubic vector fields symmetric with respect to a center, J. Differential Equations 123(2) (1995), 388-436. DOI: 10.1006/jdeq.1995.1168.

[50] C. Rousseau, D. Schlomiuk, and P. Thibaudeau, The centres in the reduced Kukles system, Nonlinearity 8(4) (1995), 541-569. DOI : $10.1088 / 0951-7715 / 8 / 4 / 005$.

[51] D. Schlomiuk, Algebraic particular integrals, integrability and the problem of the center, Trans. Amer. Math. Soc. 338(2) (1993), 799-841.

[52] D. Schlomiuk, J. Guckenheimer, And R. Rand, Integrability of plane quadratic vector fields, Exposition. Math. 8(1) (1990), $3-25$.

[53] D. Schlomiuk And J. PAL, On the geometry in the neighborhood of infinity of quadratic differential systems with a weak focus, Qual. Theory Dyn. Syst. 2(1) (2001), 1-43. DOI: $10.1007 /$ BF02969379.

[54] D. Schlomiuk And N. Vulpe, Planar quadratic vector fields with invariant lines of total multiplicity at least five, Qual. Theory Dyn. Syst. 5(1) (2004), 135-194. DOI: 10.1007/BF02968134.

[55] D. Schlomiuk And N. Vulpe, Geometry of quadratic differential systems in the neighborhood of infinity, J. Differential Equations 215(2) (2005), 357-400. DOI: 10.1016/j.jde.2004.11.001.

[56] D. Schlomiuk And N. Vulpe, Integrals and phase portraits of planar quadratic differential systems with invariant lines of at least five total multiplicity, Rocky Mountain J. Math. 38(6) (2008), 2015-2075. DOI : 10.1216/RMJ-2008-38-6-2015. 
[57] D. Schlomiuk and N. Vulpe, Planar quadratic differential systems with invariant straight lines of total multiplicity four, Nonlinear Anal. 68(4) (2008), 681-715. DOI: 10.1016/j.na.2006.11.028.

[58] D. SChlomiuk and N. VulPe, Integrals and phase portraits of planar quadratic differential systems with invariant lines of total multiplicity four, Bul. Acad. Ştiinţe Repub. Mold. Mat. 2008(1) (2008), 27-83.

[59] D. Schlomiuk and N. Vulpe, The full study of planar quadratic differential systems possessing a line of singularities at infinity, J. Dynam. Differential Equations 20(4) (2008), 737-775. DOI : $10.1007 / \mathrm{s} 10884-008-9117-2$.

[60] D. Schlomiuk and N. Vulpe, Bifurcation diagrams and moduli spaces of planar quadratic vector fields with invariant lines of total multiplicity four and having exactly three real singularities at infinity, Qual. Theory Dyn. Syst. 9(1-2) (2010), 251-300. DOI : $10.1007 / \mathrm{s} 12346-010-0028-3$.

[61] D. Schlomiuk And N. Vulpe, Global classification of the planar Lotka-Volterra differential systems according to their configurations of invariant straight lines, J. Fixed Point Theory Appl. 8(1) (2010), 177-245. DOI : $10.1007 / \mathrm{s} 11784-010-0031-\mathrm{y}$.

[62] D. Schlomiuk and N. Vulpe, Global topological classification of Lotka-Volterra quadratic differential systems, Electron. J. Differential Equations 2012(64) (2012), 69 pp.

[63] H. Seifert And W. Threlfall, "Seifert and Threlfall: a textbook of topology", Translated from the German edition of 1934 by Michael A. Goldman, Pure and Applied Mathematics 89, Academic Press, Inc. [Harcourt Brace Jovanovich, Publishers], New York-London, 1980.

[64] K. S. SiBinsky, "Introduction to the algebraic theory of invariants of differential equations", Translated from the Russian, Nonlinear Science: Theory and Applications, Manchester University Press, Manchester, 1988.

[65] C. H. Taubes, Differential topology, in: "The Princeton Companion to Mathematics" (T. Gowers, J. Barrow-Green and I. Leader, eds.), Princeton University Press, Princeton, NJ, 2008, pp. 396-408.

[66] M. Voldman, Iu. T. Calin, and N. I. Vulpe, Affine invariant conditions for the topological distinction of quadratic systems with a critical point of the 4th multiplicity, Publ. Mat. 40(2) (1996), 431-441. DOI: 10.5565/PUBLMAT_40296_13.

[67] M. Voldman and N. I. Vulpe, Affine invariant conditions for topologically distinguishing quadratic systems without finite critical 
points, Izv. Akad. Nauk Respub. Moldova Mat. 1995(2-3) (1995), 100-112, 114, 117.

[68] M. Voldman and N. I. Vulpe, Affine invariant conditions for topologically distinguishing quadratic systems with $m_{f}=1$, Nonlinear Anal. 31(1-2) (1998), 171-179. DOI: 10.1016/S0362-546X (96) 00302-1.

[69] N. I. Vulpe, Affine-invariant conditions for topological distinction of quadratic systems in the presence of a center, (Russian), Differentsial'nye Uravneniya 19(3) (1983), 371-379.

[70] N. I. VulPE, "Polynomial bases of comitants of differential systems and their applications in qualitative theory", (Russian), With English and French summaries, "Shtiintsa", Kishinev, 1986. 172 pp.

[71] N. I. Vulpe And A. Yu. Likhovetskil, Coefficient conditions for the topological discrimination of quadratic systems of Darboux type, (Russian), Mat. Issled. 106, Differ. Uravneniya i Mat. Fizika (1989), 34-49, 178.

[72] N. I. Vulpe And I. V. Nikolaev, Topological classification of QS with a unique third order singular point, Izv. Akad. Nauk Respub. Moldova Mat. 1992(2) (1992), 37-44.

[73] N. I. Vulpe And I. V. Nikolaev, Topological classification of a quadratic system with a four-fold singular point, (Russian), Differentsial'nye Uravneniya 29(10) (1993), 1669-1674, 1836; translation in: Differential Equations 29(10) (1993), 1449-1453 (1994).

[74] N. I. Vulpe And K. S. Sibirskil, Affinely invariant coefficient conditions for the topological distinctness of quadratic systems, (Russian), Mat. Issled. 10, no. 3(37) (1975), 15-28, 238.

[75] N. I. Vulpe AND K. S. SibIRSKIĬ, Geometric classification of a quadratic differential system, (Russian), Differencial'nye Uravnenija 13(5) (1977), 803-814, 963.

[76] H. ŻoŁA̧DEK, Quadratic systems with center and their perturbations, J. Differential Equations 109(2) (1994), 223-273. DOI: 10.1006/jdeq.1994.1049.

Département de Mathématiques et de Statistique

Université de Montréal

Montréal QC H3C 3J7

Canada

E-mail address: dasch@dms.umontreal.ca 